\title{
Prednisolone adverse events in the treatment and prevention of leprosy neuropathy in two large double blind randomized clinical trials
}

Erik Post $^{\mathrm{a}}$, Inge Wagenaar ${ }^{\mathrm{b}}$, Wim Brandsma ${ }^{\mathrm{c}}$, Bob Bowers ${ }^{\mathrm{d}}$, Khorshed Alam ${ }^{\mathrm{d}}$, Vanaja Shetty ${ }^{\mathrm{e}}$, Vivek Pai ${ }^{\mathrm{f}}$, Sajid Husain ${ }^{\mathrm{g}}$, Cita Rosita Sigit Prakoeswa ${ }^{\text {h }}$, Linda Astari ${ }^{\text {h }}$, Deanna Hagge ${ }^{\mathrm{i}}$, Mahesh Shah ${ }^{i}$, Kapil Neupane ${ }^{\mathrm{i}}, \mathrm{Krishna} \mathrm{Lama}^{\mathrm{j}}$, Peter Nicholls ${ }^{\mathrm{k}}$ \& Jan Hendrik Richardus ${ }^{\mathrm{b}}$

${ }^{a}$ Royal Tropical Institute, KIT Health, Amsterdam, The Netherlands

${ }^{\mathrm{b}}$ Department of Public Health, Erasmus University Medical Center, Rotterdam, The Netherlands

${ }^{\mathrm{c}}$ Independent Leprosy Consultant, Royal Tropical Institute, Amsterdam, The Netherlands

${ }^{\mathrm{d}}$ Rural Health Program, The Leprosy Mission International Bangladesh, Nilphamari, Bangladesh

${ }^{\mathrm{e}}$ Foundation for Medical Research, Mumbai, India

${ }^{\mathrm{f}}$ Bombay Leprosy Project, Mumbai, India

${ }^{\mathrm{g}}$ JALMA institute of Leprosy \& Other Mycobacterial Diseases, Agra, India

${ }^{\mathrm{h}}$ Dr Soetomo Teaching Hospital - Universitas Airlangga, Surabaya,

Indonesia

${ }^{\mathrm{i}}$ Anandaban Hospital and Mycobacterial Research Laboratories, The

Leprosy Mission Nepal, Kathmandu, Nepal

${ }^{\mathrm{j}}$ Lalgadh Leprosy Hospital and Services Centre Dhanusha, Lalgadh, Nepal

${ }^{\mathrm{k}}$ Faculty of Health Sciences, University of Southampton, Southampton, UK

Submitted 21 June 2021; Accepted 30 June 2021

\begin{abstract}
Summary
Objectives To document serious adverse events (SAE) of prolonged prednisolone use in two double-blind randomized clinical trials (RCT). In the first RCT, treatment effectiveness on restoring nerve function of a 20 -week prednisolone regimen was compared with a 32-week course. In the second RCT, patients with subclinical neuropathy received either 20 weeks prednisolone or placebo to assess whether prednisolone could prevent the development of clinical neuropathy. In both trials, patients received a
\end{abstract}

Correspondence to: Erik Post, Royal Tropical Institute, KIT Health, Amsterdam, The Netherlands (e-mail: erikbaspost@gmail.com)

See acknowledgement for names of the members of the study group.

(c) The author(s). This article is Open Access under CC BY 4.0 
prednisolone starting dose according to weight: high weight ( $\geq 50 \mathrm{~kg}$ ) started at $60 \mathrm{mg}$ and low weight $(<50 \mathrm{~kg})$ at $45 \mathrm{mg}$. In both trials, the occurrence of serious adverse events (SAE) was an important secondary outcome.

Results In the first RCT, 868 patients were enrolled, of whom $16(1.8 \%)$ developed a serious adverse event (SAE). There were 12 SAEs in the longer treatment arm $(N$ $=439$, event rate of $2.7 \%)$, and four in the shorter arm $(N=429$, event rate of $0.9 \%)$ $(p=0.041)$. In the second RCT 4/364 (1.1\%) developed an SAE, of which one was in the placebo arm. In both trials, minor adverse events were quite common and varied greatly in frequency and between centres.

Conclusions When searching for the optimum dose and duration of prednisolone in the treatment and prevention of neuropathy in leprosy patients, one must weigh possible advantages against the possible serious adverse events. Although our trials showed a significantly increased percentage of SAE in the longer treatment arm in the first RCT and in the treatment arm of the second RCT, the overall event rate was very low.

Keywords: Leprosy reactions, prednisolone, adverse effects

\section{Introduction}

Leprosy is a disease of skin and nerves. Leprosy neuropathy is a major complication that may lead to permanent disability when untreated. Nerve function impairment (NFI) can be prevented if neuropathy is diagnosed in time and the patient is given a course of an immunosuppressive drug, such as a prednisolone. ${ }^{1-4}$

The World Health Organization has recommended regimens for the treatment of neuropathy taking into account age (child/adult) and type of "reactional" neuropathy, typically a 12-weeks regimen. ${ }^{5}$ In case of a type 1 reaction, ILEP advises a 12-weeks regimen for PB cases, and a 24-weeks regimen for MB cases. For type 2 reaction, this is a short course of 6 weeks. ${ }^{6}$

Many studies have shown both benefits and limitations of treating leprosy neuropathy with corticosteroids using different treatment regimens, with variations in dose and duration. ${ }^{1-3,7}$ These studies have shown that treatment durations longer than proposed in the WHO guideline are more effective.

Outside the field of leprosy, the adverse events of corticosteroids have been well documented for the treatment of diverse pathologies. ${ }^{8}$

In a multi-centre randomised, double blind clinical trial the effectiveness of a longer, 32 weeks course of prednisolone was compared with the commonly accepted treatment regimens of 20 weeks in improving and restoring clinically established recent sensory and/or motor loss (from now on called the clinical trial). ${ }^{9}$

In a parallel randomised, double-blind and placebo-controlled trial, prednisolone was given for 20 weeks to patients who had only subclinical nerve impairment, as assessed by nerve conduction studies (NCS) and/or Warmth Detection Thresholds (WDT) ${ }^{1}$ (from now on called the subclinical trial). ${ }^{10-12}$ The aim of the study was to determine whether prednisolone treatment would prevent clinical function loss in leprosy patients as established by Voluntary Muscle Testing (VMT) and Monofilament Testing (MFT). These two trials are known as the

\footnotetext{
${ }^{1}$ TSA-Den Medical. Dorpsstraat 149-153, 1640 Sint-Genesius-Rode, Belgium; NCS: Biotech, 15 Kamal Industrial Estate, Boriali, Mumbai 400066, India.
} 
Table 1. The types of adverse events as recorded this study

\begin{tabular}{ll}
\hline Serious adverse events (SAE) & Minor adverse events (MAE) \\
\hline Peptic ulcer & Gastric pain \\
Diabetes & Moon face \\
Tuberculosis & Acne \\
Hypertension & Fungal infection, local \\
Psychosis & Weight gain \\
Glaucoma & Other*** \\
Cataract & \\
Corneal ulcer* & \\
Worm infestation & \\
Other** & \\
${ }^{*}$ Specific for leprosy. ${ }^{* *}$ Cardiovascular events (rare), osteoporosis (screening for exclusion), adrenal insufficiency \\
(rare), myopathy (rare), other infection. ${ }^{* * *}$ At the discretion of the clinician.
\end{tabular}

TENLEP trials (Treatment of Early Neuropathy in LEProsy). ${ }^{13}$ This study reports on possible steroid induced serious and minor adverse events in the two TENLEP trials.

\section{Methods}

The trials took place in six centres in four leprosy endemic countries: India (2), Nepal (2), Bangladesh and Indonesia. Eligible patients were assessed with Voluntary Muscle Tests (VMT), Monofilament Tests (MFT), Nerve Conduction Studies (NCS), and Warmth Detection Tests (WDT). Reliability and normative studies were carried out prior to screening for possible inclusion. Patients, between the ages of 15 and 60, were eligible for enrolment, irrespective of the leprosy classification. More detailed information regarding inclusion and exclusion criteria can be found in the protocol article. ${ }^{13}$ Patients were monitored for 78 weeks, whereby assessments were carried out monthly until completion of the intervention at 20 (subclinical trial) or 32 weeks (clinical trial), followed by assessments at week 52 and week 78 .

In both trials the medication provided is prednisolone. Adverse events were recorded as listed in Table 1.

In the clinical trial, patients in the treatment arm received prednisolone for 32 weeks, in tablets of $5 \mathrm{mg} .{ }^{13}$ The control arm followed a regimen of 20 weeks and received placebo tablets to keep the number of tablets equal to that in the treatment group. The dose in both intervention and control arm started at on average $1 \mathrm{mg} / \mathrm{kg} /$ day (either 45 or $60 \mathrm{mg} /$ day depending on body weight being under or above $50 \mathrm{~kg}$ respectively) and was tapered down over 32 and 20 weeks, respectively (see Figure 1). In contrast to previous trials, the middle range of the prednisolone dose was maintained at a high level $(0.44 \mathrm{mg} / \mathrm{kg} / \mathrm{day})$ for a longer period (12 weeks).

In the subclinical trial, patients received either prednisolone or placebo for 20 weeks in tablets of $5 \mathrm{mg}$. The prednisolone dose started at $1 \mathrm{mg} / \mathrm{kg} /$ day (either $45 \mathrm{or} 60 \mathrm{mg} /$ day depending on weight class), and was be tapered down over 20 weeks. Figure 1 shows the timeline and dosage. The total dosage of prednisolone over 20 weeks will be $2.8 \mathrm{~g}$ for patients under $50 \mathrm{~kg}$ body weight, and $3.7 \mathrm{~g}$ for patients over $50 \mathrm{~kg}$ body weight.

Prior to enrolment, patients were screened for underlying morbidities such as diabetes and osteoporosis, amongst others. When detected, these patients were excluded from the trials. All patients were de-wormed before starting the trial medication.

A distinction was made between minor and serious adverse events. Adverse events were only counted once, even when they occurred multiple times in one patient or were recorded on 

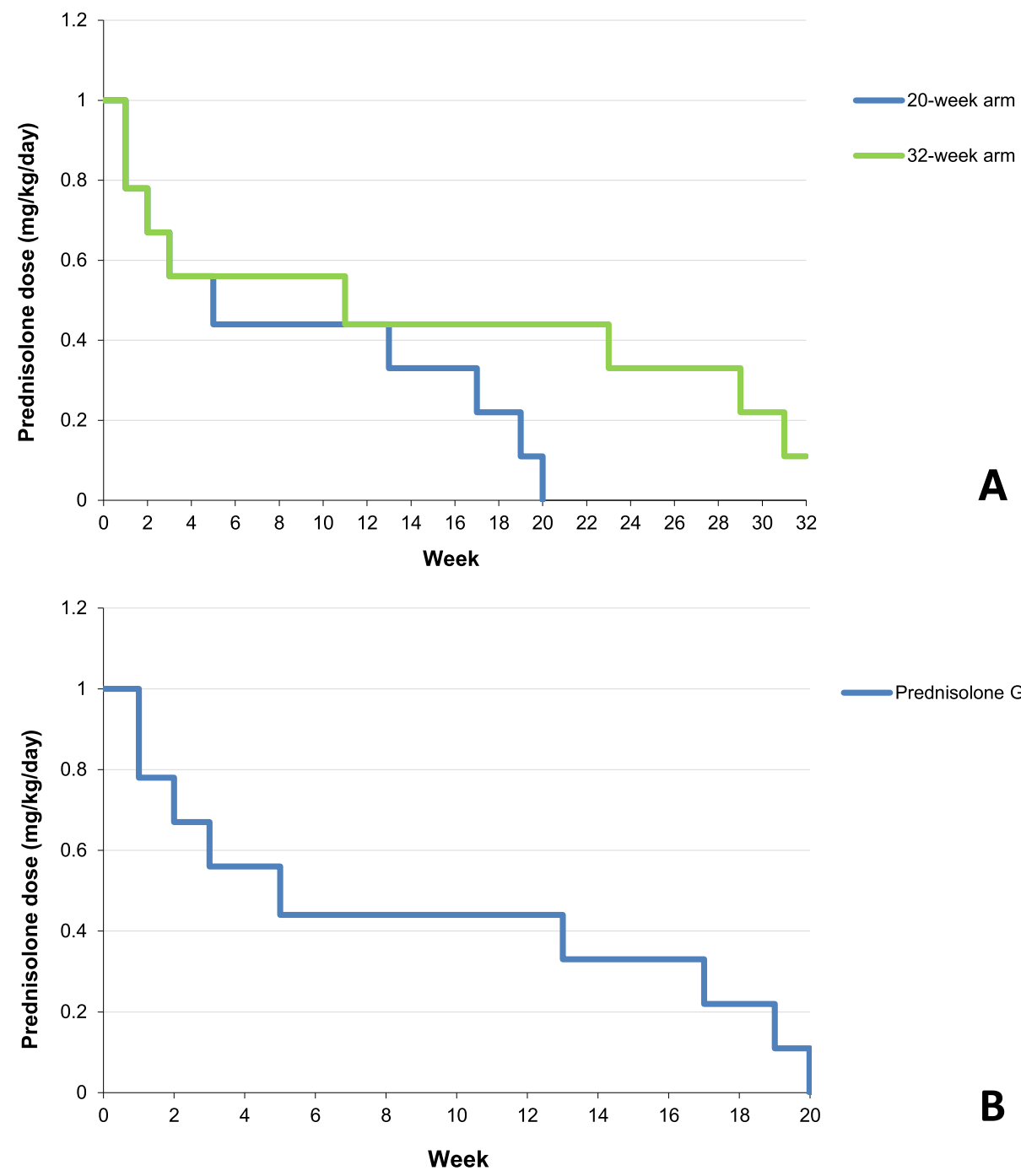

Prednisolone Group

Figure 1. (A) Timeline prednisolone dose for patients under $50 \mathrm{~kg}$ in the Clinical trial. (B) Timeline prednisolone dose for patients under $50 \mathrm{~kg}$ in the Subclinical trial.

multiple visits. When minor adverse events were noticed these were treated and the patient continued in the trial. When serious adverse events were noticed or leprosy reactions or other complications occurred, patients were taken off the trial treatment and continued on individualised treatment.

Data from the participating centres were sent to one author (PN) and were analysed with Stata.

Informed written consent was obtained from each patient, who could withdraw from the treatment at any time. In India, ethics approval was obtained from the Indian Council of Medical Research and the Ethics Committee of the Foundation for Medical Research, Mumbai; in Nepal from the Nepal Health Research Council (NHRC 14/2011); in Indonesia from the 
Table 2. Relative Risk, Confidence Intervals (CI) and $p$-values of adverse events in both trials

\begin{tabular}{lccccc}
\hline Clinical trial & $\begin{array}{c}20 \text {-week arm } \\
n=429\end{array}$ & $\begin{array}{c}\text { 32-week arm } \\
n=439\end{array}$ & RR & CI & $p_{\text {-value }}^{*}$ \\
\hline $\begin{array}{l}\text { Serious adverse events } \\
\text { Minor adverse events }\end{array}$ & 4 & 12 & 2.9 & $(0.95-9.02)$ & 0.041 \\
& 284 & 300 & 1.0 & $(0.94-1.13)$ & 0.275 \\
Subclinical trial & 20-week arm & Placebo & RR & CI & $p$-value* \\
& $n=181$ & $n=182$ & & & 0.309 \\
Serious adverse events & 3 & 1 & 3.0 & $(0.32-28.73)$ & 0.003 \\
Minor adverse events & 102 & 75 & 1.4 & $(1.10-1.70)$ &
\end{tabular}

Table 3. Serious adverse events in Clinical trial

\begin{tabular}{|c|c|c|c|c|c|c|c|c|c|c|}
\hline \multirow[t]{2}{*}{ Centre } & \multicolumn{5}{|c|}{ 20-week arm } & \multicolumn{5}{|c|}{ 32-week arm } \\
\hline & A & $\mathrm{B}$ & $\mathrm{C}$ & $\mathrm{D}$ & $\mathrm{E}$ & $\mathrm{A}$ & B & $\mathrm{C}$ & $\mathrm{D}$ & $\mathrm{E}$ \\
\hline Peptic ulcer & & & & $1^{\dagger}$ & & & $1^{*}$ & & & \\
\hline $\begin{array}{l}\text { Diabetes } \\
\text { Tuberculosis }\end{array}$ & & & & $1^{*}$ & $1^{*}$ & & & $1^{*}$ & $3^{\dagger * *}$ & \\
\hline $\begin{array}{l}\text { Hypertension } \\
\text { Psychosis } \\
\text { Other }\end{array}$ & & & & $1^{*}$ & & $1^{*}$ & & $\begin{array}{c}2^{\dagger *} \\
1^{*}\end{array}$ & $\begin{array}{c}2^{\dagger \dagger} \\
1^{*}\end{array}$ & \\
\hline
\end{tabular}

Centre F did not report any SAE; ${ }^{\dagger}$ In low weight group; ${ }^{*}$ In high weight group.

Komite Etik Penelitian Kesehatan RSUD Dr. Soetomo Surabaya; in Bangladesh from the Bangladesh Medical Research Council - National Research Ethics Committee. The trial was registered in the Netherlands Trial Register (NTR2300) and in the Clinical Trials registry India (CTRI/2011/09/002022 and 23).

\section{Results}

A total of 868 patients were enrolled in the clinical trial and 364 patients in the subclinical trial. In both trials there were no statistically significant differences in demographics nor assignment to the treatment arms. ${ }^{9,14}$

Table 2 summarises the serious and minor adverse events from both trials. In the subclinical trial, with a prednisolone ( 20 weeks) and a placebo arm, four SAEs were noticed: three patients with hypertension and one patient with TB, the latter who had been assigned to the placebo arm. All SAEs in the subclinical trial were reported by one centre, and all were in the high weight group.

In the clinical trial, with two treatment arms, 16 SAE were recorded. Table 3 shows the SAEs per centre and trial arm. It is noticeable that not all possible SAEs, as listed in Table 1, occurred in our cohort. In addition, it can be seen that there is considerable variation between the centres in the number of SAEs reported. All SAEs were reported within the period that prednisolone was given. More SAEs were reported from the longer treatment arm, namely 12 of the $16(p=0.041)$. Of these 16,11 were in the high weight group $(p=0.378)$. 
Table 4. Minor Adverse Events in Subclinical Trial per treatment arm and per centre

\begin{tabular}{|c|c|c|c|c|c|c|c|c|}
\hline & \multirow[t]{2}{*}{$n$ (total) } & \multirow[t]{2}{*}{$n$ (20-week arm) } & \multicolumn{2}{|c|}{ 20-week arm } & \multicolumn{2}{|c|}{ Placebo arm } & \multicolumn{2}{|c|}{ Total } \\
\hline & & & $n$ & $\%$ & $n$ & $\%$ & $n$ & $\%$ \\
\hline \multicolumn{9}{|c|}{ Moon face } \\
\hline A & 118 & 59 & 3 & $(5 \%)$ & 1 & $(2 \%)$ & 4 & $(3 \%)$ \\
\hline B & 136 & 68 & 56 & $(82 \%)$ & 36 & $(53 \%)$ & 92 & $(68 \%)$ \\
\hline $\mathrm{C}$ & 61 & 30 & 1 & $(3 \%)$ & 0 & $(0 \%)$ & 1 & $(2 \%)$ \\
\hline $\mathrm{D}$ & 48 & 24 & 4 & $(17 \%)$ & 0 & $(0 \%)$ & 4 & $(8 \%)$ \\
\hline \multicolumn{9}{|c|}{ Acne } \\
\hline A & 118 & 59 & 7 & $(12 \%)$ & 3 & $(5 \%)$ & 10 & $(8 \%)$ \\
\hline B & 136 & 68 & 52 & $(76 \%)$ & 31 & $(46 \%)$ & 83 & $(61 \%)$ \\
\hline $\mathrm{C}$ & 61 & 30 & 3 & $(10 \%)$ & 2 & $(6 \%)$ & 5 & $(8 \%)$ \\
\hline $\mathrm{D}$ & 48 & 24 & 5 & $(21 \%)$ & 1 & $(4 \%)$ & 6 & $(13 \%)$ \\
\hline \multicolumn{9}{|c|}{ Fungal infection } \\
\hline A & 118 & 59 & 3 & $(5 \%)$ & 1 & $(2 \%)$ & 4 & $(3 \%)$ \\
\hline B & 136 & 68 & 8 & $(12 \%)$ & 4 & $(6 \%)$ & 12 & $(9 \%)$ \\
\hline $\mathrm{C}$ & 61 & 30 & 1 & $(3 \%)$ & 2 & $(6 \%)$ & 3 & $(5 \%)$ \\
\hline $\mathrm{D}$ & 48 & 24 & 1 & $(4 \%)$ & 2 & $(8 \%)$ & 3 & $(6 \%)$ \\
\hline \multicolumn{9}{|c|}{ Gastric pain } \\
\hline A & 118 & 59 & 19 & $(32 \%)$ & 21 & $(36 \%)$ & 40 & $(34 \%)$ \\
\hline B & 136 & 68 & 7 & $(10 \%)$ & 4 & $(6 \%)$ & 11 & $(8 \%)$ \\
\hline $\mathrm{C}$ & 61 & 30 & 0 & $(0 \%)$ & 0 & $(0 \%)$ & 0 & $(0 \%)$ \\
\hline $\mathrm{D}$ & 48 & 24 & 0 & $(0 \%)$ & 0 & $(0 \%)$ & 0 & $(0 \%)$ \\
\hline \multicolumn{9}{|c|}{ Weight gain } \\
\hline A & 118 & 59 & 6 & $(10 \%)$ & 6 & $(10 \%)$ & 12 & $(10 \%)$ \\
\hline $\mathrm{B}$ & 136 & 68 & 18 & $(26 \%)$ & 12 & $(18 \%)$ & 30 & $(22 \%)$ \\
\hline $\mathrm{C}$ & 61 & 30 & 3 & $(10 \%)$ & 0 & $(0 \%)$ & 3 & $(5 \%)$ \\
\hline $\mathrm{D}$ & 48 & 24 & 1 & $(4 \%)$ & 0 & $(0 \%)$ & 1 & $(2 \%)$ \\
\hline \multicolumn{9}{|c|}{ Other } \\
\hline A & 118 & 59 & 1 & $(2 \%)$ & 2 & $(3 \%)$ & 3 & $(3 \%)$ \\
\hline B & 136 & 68 & 0 & $(0 \%)$ & 0 & $(0 \%)$ & 0 & $(0 \%)$ \\
\hline $\mathrm{C}$ & 61 & 30 & 0 & $(0 \%)$ & 0 & $(0 \%)$ & 0 & $(0 \%)$ \\
\hline $\mathrm{D}$ & 48 & 24 & 0 & $(0 \%)$ & 0 & $(0 \%)$ & 0 & $(0 \%)$ \\
\hline
\end{tabular}

The minor adverse events are reported in Tables 4 and 5. In both trials, minor adverse events were very common: $49 \%$ of the patients in the subclinical trial and $67 \%$ of the patients in the clinical trial showed one or more MAEs. MAEs varied greatly in occurrence of event and between centres: for example, in the subclinical trial: moon face (2-68\%), and acne (8-61\%). Minor events were also noticed in the placebo arm: for example, moon face: 0-53\% (Table 4). The same great variation in numbers of adverse events between the centres is also noticeable for the clinical trial (Table 5).

In both trials and all arms, differences could be seen between the high and low weight groups; however, the differences were not clearly in one direction. Of the high weight group in the subclinical trial $44 \%$ had an MAE and in the clinical trial $66 \%$. In the low weigh groups, this was $56 \%$ and $69 \%$, respectively. 
Table 5. Minor Adverse Events in Clinical Trial per treatment arm and per centre

\begin{tabular}{|c|c|c|c|c|c|c|c|c|}
\hline & \multirow[t]{2}{*}{$n$ (total) } & \multirow[t]{2}{*}{$n$ (20-week arm) } & \multicolumn{2}{|c|}{ 20-week arm } & \multicolumn{2}{|c|}{ 32-week arm } & \multicolumn{2}{|c|}{ Total } \\
\hline & & & $n$ & $\%$ & $n$ & $\%$ & $n$ & $\%$ \\
\hline \multicolumn{9}{|c|}{ Moon face } \\
\hline A & 82 & 40 & 3 & $(8 \%)$ & 1 & $(2 \%)$ & 4 & $(5 \%)$ \\
\hline B & 230 & 115 & 99 & $(86 \%)$ & 97 & $(84 \%)$ & 196 & $(84 \%)$ \\
\hline $\mathrm{C}$ & 166 & 83 & 12 & $(14 \%)$ & 11 & $(13 \%)$ & 23 & $(14 \%)$ \\
\hline $\mathrm{D}$ & 232 & 116 & 24 & $(21 \%)$ & 31 & $(27 \%)$ & 55 & $(24 \%)$ \\
\hline $\mathrm{E}$ & 101 & 48 & 13 & $(27 \%)$ & 15 & $(28 \%)$ & 28 & $(28 \%)$ \\
\hline $\mathrm{F}$ & 57 & 27 & 23 & $(85 \%)$ & 29 & $(97 \%)$ & 52 & $(91 \%)$ \\
\hline \multicolumn{9}{|c|}{ Acne } \\
\hline A & 82 & 40 & 7 & $(18 \%)$ & 6 & $(14 \%)$ & 13 & $(16 \%)$ \\
\hline B & 230 & 115 & 98 & $(85 \%)$ & 97 & $(84 \%)$ & 195 & $(85 \%)$ \\
\hline $\mathrm{C}$ & 166 & 83 & 14 & $(17 \%)$ & 15 & $(18 \%)$ & 29 & $(17 \%)$ \\
\hline $\mathrm{D}$ & 232 & 116 & 15 & $(13 \%)$ & 22 & $(19 \%)$ & 37 & $(16 \%)$ \\
\hline $\mathrm{E}$ & 101 & 48 & 20 & $(42 \%)$ & 15 & $(28 \%)$ & 35 & $(35 \%)$ \\
\hline $\mathrm{F}$ & 57 & 27 & 7 & $(26 \%)$ & 8 & $(27 \%)$ & 15 & $(26 \%)$ \\
\hline \multicolumn{9}{|c|}{ Fungal infection } \\
\hline A & 82 & 40 & 0 & $(0 \%)$ & 0 & $(0 \%)$ & 0 & $(0 \%)$ \\
\hline B & 230 & 115 & 11 & $(10 \%)$ & 15 & $(13 \%)$ & 26 & $(11 \%)$ \\
\hline $\mathrm{C}$ & 166 & 83 & 4 & $(5 \%)$ & 11 & $(13 \%)$ & 15 & $(9 \%)$ \\
\hline $\mathrm{D}$ & 232 & 116 & 24 & $(21 \%)$ & 28 & $(24 \%)$ & 52 & $(22 \%)$ \\
\hline $\mathrm{E}$ & 101 & 48 & 13 & $(27 \%)$ & 20 & $(38 \%)$ & 33 & $(33 \%)$ \\
\hline $\mathrm{F}$ & 57 & 27 & 6 & $(22 \%)$ & 6 & $(20 \%)$ & 12 & $(21 \%)$ \\
\hline
\end{tabular}

Gastric pain

$\begin{array}{lrrrcrrrr}\text { A } & 82 & 40 & 0 & (0 \%) & 0 & (0 \%) & 0 & (0 \%) \\ \text { B } & 230 & 115 & 19 & (17 \%) & 14 & (12 \%) & 33 & (14 \%) \\ \text { C } & 166 & 83 & 0 & (0 \%) & 0 & (0 \%) & 0 & (0 \%) \\ \text { D } & 232 & 116 & 54 & (47 \%) & 51 & (44 \%) & 105 & (45 \%) \\ \text { E } & 101 & 48 & 16 & (33 \%) & 15 & (28 \%) & 31 & (31 \%) \\ \text { F } & 57 & 27 & 7 & (26 \%) & 11 & (37 \%) & 18 & (32 \%)\end{array}$

Weight gain

$\begin{array}{lrrrrrrrr}\mathrm{A} & 82 & 40 & 0 & (0 \%) & 1 & (2 \%) & 1 & (1 \%) \\ \mathrm{B} & 230 & 115 & 64 & (56 \%) & 65 & (57 \%) & 129 & (56 \%) \\ \mathrm{C} & 166 & 83 & 1 & (1 \%) & 1 & (1 \%) & 2 & (1 \%) \\ \mathrm{D} & 232 & 116 & 54 & (47 \%) & 61 & (53 \%) & 115 & (50 \%) \\ \mathrm{E} & 101 & 48 & 26 & (54 \%) & 39 & (74 \%) & 65 & (64 \%) \\ \mathrm{F} & 57 & 27 & 20 & (74 \%) & 25 & (83 \%) & 45 & (79 \%)\end{array}$

\begin{tabular}{lrrrrrrrr} 
Other & & & & & & \\
$\mathrm{A}$ & 82 & 40 & 1 & $(3 \%)$ & 1 & $(2 \%)$ & 2 & $(2 \%)$ \\
$\mathrm{B}$ & 230 & 115 & 5 & $(4 \%)$ & 7 & $(6 \%)$ & 12 & $(5 \%)$ \\
$\mathrm{C}$ & 166 & 83 & 0 & $(0 \%)$ & 0 & $(0 \%)$ & 0 & $(0 \%)$ \\
$\mathrm{D}$ & 232 & 116 & 4 & $(3 \%)$ & 4 & $(3 \%)$ & 8 & $(3 \%)$ \\
$\mathrm{E}$ & 101 & 48 & 0 & $(0 \%)$ & 0 & $(0 \%)$ & 0 & $(0 \%)$ \\
$\mathrm{F}$ & 57 & 27 & 1 & $(4 \%)$ & 0 & $(0 \%)$ & 1 & $(2 \%)$ \\
\hline
\end{tabular}




\section{Discussion}

The TENLEP trials were conducted to (a) assess the effectiveness of a relatively longer course of prednisolone than normally practiced in the treatment of recent NFI and (b) to assess if a 20-week course of prednisolone would reduce the incidence of clinical NFI in patients with subclinical neuropathy. ${ }^{13}$ The results of these trials are reported elsewhere. ${ }^{9,14}$ An important secondary outcome was the occurrence of adverse events due to prednisolone. Do hypothesised benefits outweigh the possible increase in incidence of prednisolone related serious adverse events?

The clinical trial did not show a significant beneficial effect of 32 weeks prednisolone in the treatment of NFI compared to the "standard" 20-week course. Likewise, the treatment in the subclinical trial was not significantly more effective than a placebo in the prevention of NFI.

Our studies have shown that serious adverse events do occur, but that the overall event rate of SAE was very low. A longer duration of prednisolone (32 weeks), however, did significantly increase the event rate of SAEs.

It should be noted that patients in our clinical neuropathy trial not only received prednisolone for a longer duration then commonly practiced in leprosy, also the total amount of prednisolone was higher. This was not only because of the longer duration, but also because we maintained a "middle-medium plateau" dose for a relative longer time before tapering down (Figure 1). The plateau dose remained above the "critical" dose of $25 \mathrm{mg}$ as recommended by Naafs except for the last three weeks. ${ }^{1}$ Despite the somewhat higher dose used in our trials, SAE event rates were quite similar at to those in the TRIPOD trials, at less than $3 \% .{ }^{15}$ All patients developing SAE did so during the trial treatment period of 32 weeks. In both trials, when patients had deteriorating nerve function or reactions, they received additional prednisolone. None of those patients developed SAE.

In one of the centres, many patients were recalled for an additional physical examination after the 72 weeks follow up period, but within 120 weeks after enrolment in the trial. An additional three patients were diagnosed with cataract and two with hypertension. Hypertension can have multiple causes and is not likely to be related to the use of prednisone many months previously.

Regarding cataract, there seems to be a relationship between prednisolone treatment and a specific type of cataract, sub-capsular. This specific type was diagnosed in two of the patients. The aetiology of steroid induced posterior subcapsular cataracts is explained by James. ${ }^{16}$ One study in lepromatous leprosy patients in India found that cataract develops in 3\% per person years at risk and $7 \%$ per person years at risk in lepromatous patients over 40 years of age. There was, however, no significant association with oral steroids. Leprosy patients are "at risk" because of the generally long duration of the disease and often present subclinical intraocular inflammation. ${ }^{2,17}$ Further prospective studies are recommended to investigate the late occurrence of cataract and its possible relation to prednisolone.

Minor adverse events have been reported in another placebo controlled trial, and similar to our study, the MAEs were present in the placebo arm, though less frequently. ${ }^{15}$ It appears, therefore, that some of the minor adverse events that can be attributed to prednisolone also happen due to other causes, co-incidentally. Some, like moon face, depend on subjective clinical judgement. MAEs can be treated or will resolve when prednisolone treatment is stopped. However, they can be aesthetically unacceptable and therefore need careful explanation. ${ }^{18}$

This study had some limitations to take into consideration. First, even though the trial was double-blind, experienced leprosy physicians and field-staff may suspect that patients are on the prednisolone arm through, for example, the appearance of moon-face features, or acne, 
which happens in many patients. ${ }^{3}$ This knowledge could influence the clinical observation, besides alertness and conscientiousness, in the recording of minor adverse events. However, this seems to be contradicted by the finding that minor adverse event were also noticed in the placebo group, confirming findings by others. ${ }^{15}$ Second, in both arms of the clinical trial there was a considerable proportion of patients who were lost to follow-up. This was seen in both the 20 -week $(96 / 429(22.4 \%)$ and the 32-week $(110 / 439=25.1 \%)$ prednisolone arms. One possible reason for losing patients to follow-up is side effects of the treatment, and therefore potentially the number of SAE could have been higher than reported. ${ }^{8,19}$

Obvious differences are seen in the incidence of minor adverse events between the project sites and one could also wonder whether these could be attributed to ethnic differences and/ or differences in alertness in noticing and recording of (minor) adverse events. ${ }^{20}$ It should be born in mind that the studies took place in six projects in four countries with structurally differing leprosy programs. Differences in services, illness behaviour, changes in staff and commitment may have a different impact on therapy adherence, noticing and reporting of especially minor adverse events, besides the possible contribution of ethnicity.

One "beneficial" adverse event that is known but little researched, is the dependency on prednisolone. ${ }^{21}$ Prednisolone supposedly may cause euphoria, which may result in patients requesting the drug, even purchasing prednisolone themselves. Fardet ${ }^{18}$ mentions many different mental consequences, not systematically recorded in our study and not mentioned by the clinicians involved in this study.

The search for alternative, more effective drugs continues, but none has been shown to be superior yet to prednisolone in terms of better outcomes and fewer adverse events. ${ }^{22,23}$ Hence, corticosteroids remain "first choice" in neuropathy and "reactional" phases in leprosy whether skin, nerve, or both are involved. More research is needed into the effect of prednisolone in the prevention and treatment of NFI and other concomitant signs of leprosy reactions, not only in the delineation of leprosy and patient specific characteristics that determine response but also in alternative drug regimens.

The TENLEP studies are two of very few studies that have looked systematically in randomized trials into the adverse events of corticosteroids in the treatment of leprosy neuropathy. Outside leprosy, adverse events due to corticosteroids have been extensively reviewed by Fardet for many other clinical conditions. ${ }^{8,18}$ Because of its completeness and clinical grouping, the classification of adverse events as developed by Fardet seems most suitable to follow in future leprosy clinical projects using steroids or other immune-suppressive drugs. ${ }^{8,24}$

Though it is important to have a protocol, or guideline document, for the treatment of nerve function impairment, ideally treatment for reaction/NFI should be patient centred. This is not always practical under field conditions with integrated services and declining leprosy expertise. Nevertheless, ideally, "treatment should be tailored to the individual patient. ... response carefully assessed and adapted to changing circumstances, immediately if necessary". ${ }^{25}$

When searching for the optimum dose and duration of prednisolone in the treatment and prevention of neuropathy in leprosy patients, one must weigh possible advantages of prednisolone treatment against the possible serious adverse events. The documented advantages of a 20-week regiment of prednisolone for the treatment of recent nerve function impairment, to a reported $70-80 \%$ improvement, outweigh the disadvantages of SAEs, especially with the low rates in our study. 


\section{Members of the TENLEP study group}

Kallyan Kumar Kunda ${ }^{1}$, Anil Soren ${ }^{1}$, Kolpona Roy ${ }^{1}$, Gospi Capadia ${ }^{2}$, Ashish Khodke ${ }^{2}$, Kulbushan Pawar ${ }^{2}$, Mishra Nath Kameshwar ${ }^{3}$, Bipin Kumar ${ }^{3}$, Hemant Singh $^{3}$, Bagus Haryo Kusumaputra $^{4}$, Irmadita Citrashanty ${ }^{4}$, Sylvia Anggraeni ${ }^{4}$, Menul Ayu Umborowati ${ }^{4}$, Renata Mayangsari $^{4}$, Riski Haris Sasongko, Agustina ${ }^{4}$, Asmahani Thohiroh ${ }^{4}$, Ram Babu Bista ${ }^{5}$, Pawan Parajuli ${ }^{5}$, Apsara Ghimiri ${ }^{5}$, Shyam Pariyar ${ }^{6}$, Jeevan Thapa ${ }^{6}$ and Shankar Paudyel ${ }^{6}$.

1 Rural Health Program, The Leprosy Mission International Bangladesh, Nilphamari, Bangladesh

${ }^{2}$ Foundation for Medical Research, Mumbai, India

${ }^{3}$ JALMA institute of Leprosy \& Other Mycobacterial Diseases, Agra, India

${ }^{4}$ Dr Soetomo Teaching Hospital - Universitas Airlangga, Surabaya, Indonesia

5 Anandaban Hospital and Mycobacterial Research Laboratories, The Leprosy Mission Nepal, Kathmandu, Nepal

${ }^{6}$ Lalgadh Leprosy Hospital and Services Centre Dhanusha, Lalgadh, Nepal.

\section{Acknowledgements}

We are immensely grateful to the patients that were willing to participate in this study. Also, we would like to thank the funders: The American Leprosy Mission, the German Leprosy and TB Relief Association, The Netherlands Leprosy Relief, the Ordre de Malte, and the Turing Foundation.

\section{Conflicts of interest}

All authors declare no conflict of interest.

\section{Authors' contributions}

Concept, design and literature review: EP, IW, WB

Data collection: All

Analysis and interpretation of findings: EP, IW, WB, PN

Write-up and presentation: EP, IW, WB

Approval of final version: All.

\section{References}

1 Naafs B, Pearson JM, Wheate HW. Reversal reaction: the prevention of permanent nerve damage. Comparison of short and long-term steroid treatment. Int J Lepr Other Mycobact Dis, 1979; 47(1): 7-12. [Internet].

2 Rao PS, Sugamaran DS, Richard J, Smith WC. Multi-centre, double blind, randomized trial of three steroid regimens in the treatment of type-1 reactions in leprosy. Lepr Rev, 2006; 77: 25-33. [Internet].

3 Sugumaran DS. Steroid therapy for paralytic deformities in leprosy. Int J Lepr Other Mycobact Dis, 1997; 65(3): 337-344. [Internet].

4 Croft RP, Nicholls PG, Richardus JH, Smith WCS. The treatment of acute nerve function impairment in leprosy: Results from a prospective cohort study in Bangladesh. Lepr Rev, 2000; 71(2): 154-168. [Internet] [cited 2014 Sep 1].

5 WHO. WHO Model Prescribing Information: Drugs used in Leprosy. Geneva: World Health Organization, 1998. [Internet].

6 ILEP. How to Recognise and Manage Leprosy Reactions. London: ILEP, 2002; p. 62.

7 van Veen NHJ, Nicholls PG, Smith WCS, Richardus JH. Corticosteroids for treating nerve damage in leprosy. A Cochrane review. Lepr Rev, 2009; 79: 361-371

8 Fardet L, Kassar A, Cabane J, Flahault A. Corticosteroid-induced adverse events in adults. Drug Saf, 2007; 30(10): 861-881. [Internet] [cited 2015 Aug 25]. 
9 Wagenaar I, Post E, Brandsma W, Bowers B, Alam K, Shetty V et al. Effectiveness of 32 versus 20 weeks of prednisolone in leprosy patients with recent nerve function impairment: A randomized controlled trial. PLoS Negl Trop Dis, 2017; 11: e0005952. [Internet] [cited 2017 Nov 21].

10 McKnight J, Nicholls PG, Loretta D, Desikan K V, Lockwood DNJ, Wilder-Smith EP et al. Reference values for nerve function assessments among a study population in northern India - ii: Thermal sensation thresholds. Neurol Asia, 2010; 15(1): 27-38. [Internet].

11 McKnight J, Nicholls PG, Loretta D, Desikan K V, Lockwood DNJ, Wilder-Smith EP et al. Reference values for nerve function assessments among a study population in northern India - iii: Sensory and motor nerve conduction. Neurol Asia, 2010; 15(1): 39-54.

12 van Brakel WH, Nicholls PG, Wilder-Smith EP, Das L, Barkataki P, Lockwood DN. Early diagnosis of neuropathy in leprosy-comparing diagnostic tests in a large prospective study (the INFIR cohort study). PLoSNeglTrop Dis, 2008; 2(4): e212. [Internet] [cited 2014 Sep 1].

13 Wagenaar I, Brandsma W, Post E, van Brakel W, Lockwood D, Nicholls P et al. Two randomized controlled clinical trials to study the effectiveness of prednisolone treatment in preventing and restoring clinical nerve function loss in leprosy: the TENLEP study protocols. BMC Neurol, 2012; 12(1): 159. [Internet] [cited 2013 May 14].

14 Post E, Wagenaar I, Brandsma J, Nicholls P, Shetty V, Husain S et al. A 5-month prophylactic prednisolone course is not effective in preventing clinical neuropathy in leprosy patients with subclinical neuropathy (in preparation).

15 Richardus JH, Withington SG, Anderson AM, Croft RP, Nicholls PG, van Brakel WH et al. Adverse events of standardized regimens of corticosteroids for prophylaxis and treatment of nerve function impairment in leprosy: results from the "TRIPOD” trials. Lepr Rev, 2003; 74(4): 319-327. [Internet].

16 James ER. The etiology of steroid cataract. J Ocul Pharmacol Ther, 2007; 23(5): 403-420.

17 Daniel E, Sundar Rao PSS. Evolution of vision reducing cataract in skin smear positive lepromatous patients: does it have an inflammatory basis?. Br J Ophthalmol, 2007; 91(8): 1011-1013. [Internet].

18 Fardet L, Flahault A, Kettaneh A, Tiev KP, Généreau T, Tolédano C et al. Corticosteroid-induced clinical adverse events: Frequency, risk factors and patient's opinion. Br J Dermatol, 2007; 157(1): 142-148.

19 Morin C, Fardet L. Systemic glucocorticoid therapy: risk factors for reported adverse events and beliefs about the drug. A cross-sectional online survey of 820 patients. Clin Rheumatol, 2015; 34(12): 2119-2126.

20 Wood AJJ, Zhou HH. Ethnic differences in drug disposition and responsiveness. Clin Pharmacokinet, 1991; 20: 350-373.

21 Swinburn C, Wakefield J, Newman S, Jones P. Evidence of prednisolone induced mood change ("steroid euphoria") in patients with chronic obstructive airways disease. Br J Clin Pharmacol, 1988; 26(6): 709-713. [Internet].

22 Naafs B, van Hees CLM. Leprosy type 1 reaction (formerly reversal reaction). Clin Dermatol, 2015; 34(1): 37-50. [Internet]. Available from: http://dx.doi.org/10.1016/j.clindermatol.2015.10.006.

23 Lambert SM, Alembo DT, Nigusse SD, Yamuah LK, Walker SL, Lockwood DNJ. A randomized controlled double blind trial of ciclosporin versus prednisolone in the management of leprosy patients with new type 1 reaction, in Ethiopia. PLoS Negl Trop Dis, 2016; 10(4): e0004502. [Internet] [cited 2016 Apr 6].

24 Liu D, Ahmet A, Ward L, Krishnamoorthy P, Mandelcorn ED, Leigh R et al. A practical guide to the monitoring and management of the complications of systemic corticosteroid therapy. Allergy Asthma Clin Immunol, 2013; 9(1): 30 .

25 Post E, chin-A-Lien RA, Bouman C, Naafs B, Faber WR. Leprosy in The Netherlands in the period 1970-1991. Ned Tijdschr Geneeskd, 1994; 138: 1960-1963. 